Narita, S., et al. Gamma Ray Detection and Surface Analysis on Palladium Electrode in DC Glow-like Discharge Experiment. in Tenth International Conference on Cold Fusion. 2003. Cambridge, MA: LENR-CANR.org. This paper was presented at the 10th International Conference on Cold Fusion. It may be different from the version published by World Scientific, Inc (2003) in the official Proceedings of the conference.

\title{
Gamma Ray Detection and Surface Analysis on Palladium Electrode in DC Glow-like Discharge Experiment
}

\author{
S. NARITA, H. YAMADA, A. ARAPI, N. SATO, D. KATO, M. YAMAMURA, M. ITAGAKI \\ Department of Electrical and Electronic Engineering, Iwate University, \\ Morioka 020-8551, Japan \\ E-mail:narita@dragon.elc.iwate-u.ac.jp
}

\begin{abstract}
We performed glow-like discharge experiments using deuterated palladium cathode in deuterium atmosphere to investigate the possibility of inducing low-energy nuclear reaction. Anomalous gamma ray emissions in the $80-230 \mathrm{keV}$ region were sometimes observed. It was assumed that a nuclear reaction took place during the experiment, producing short-lived radioisotopes, and these radioisotopes emitted the gamma rays in their decay processes. Elements and their isotopic abundance on the palladium cathodes were investigated by time-of-flight secondary ion mass spectrometry to find evidence of a nuclear reaction.
\end{abstract}

\section{INTRODUCTION}

The possibility of inducing low-energy nuclear reaction by the electric discharge method using Pd and other metal electrodes has been widely investigated for several years. In those experiments, many interesting results such as excess heat, new element production, and detection of radiation, have been reported numerous times [1-7]. We have reported low energy gamma ray emissions in $70-110 \mathrm{keV}$ region during DC glow discharge in $\sim 3$ Torr of deuterium gas, and element production on the surface and in the bulk of Pd electrodes using deuterium and hydrogen gas [8,9], although the phenomena were still irreproducible. In this study, we carried out a similar discharge experiment, but we increased the pressure of atmospheric gas (deuterium or hydrogen) to $\sim$ latm. We expected the reaction efficiency would be enhanced. This discharge condition was definitely different from glow discharge one, so we designated it a "glow-like" discharge. According to the energies of the emissions observed in most experiments, radioisotopes may have been produced during the experiments. However, very few high-energy particles, such as high-energy neutrons, were detected. This indicates that there is a so-called "clean" reaction in which new elements including radioisotopes are produced. In this study, we attempted to observe gamma ray emission and nuclear products by elemental analysis of Pd cathode surface with the aim of obtaining evidence of a nuclear reaction. Moreover, we tried to specify the reaction occurred in the system by analyzing the energy of the gamma rays precisely, and its transmutation process systematically.

\section{EXPERIMENT}

The experiments were carried out by DC glow-like discharge with a $\mathrm{Pd} / \mathrm{D}$ foil cathode in deuterium atmosphere. The Pd sample used was $10 \times 10 \times 0.1 \mathrm{~mm}^{3}$ in size and $>99.95 \%$ in purity. At first, each sample was washed with acetone and aqua regia for 100 s. Then, it was put in a loading chamber and loaded with deuterium gas under 10atm pressure for $\sim 48 \mathrm{~h}$. The loading ratio was determined by measuring the mass change of the sample, and it was typically 0.6-0.7. After gas loading, the sample was placed in a discharge cell shown in Figure 1 . The test cell made of Pyrex glass has a cylindrical shape with a diameter of $\sim 10 \mathrm{~cm}$ and a height of $\sim 14 \mathrm{~cm}$, which consists of two parts adhered by silicone grease (Dow Corning). The advantage of using Pyrex glass material compared with using a metal is that Pyrex minimizes 
the tendency of Pd samples to be contaminated during the sputtering process by discharge. The cell is connected to a vacuum system via a valve on the upper part of the cell. An Au foil $(0.3 \mathrm{~mm}$ in thickness) hung by an Au wire was used as the anode. The Pd sample was placed as a cathode on an Au stand with a quartz cylinder surrounding it to prevent movement during the discharge. The gap distance between two the two electrodes was $\sim 10 \mathrm{~mm}$. After closing up the cell, it was evacuated to $10^{-2}-10^{-3}$ Torr and deuterium gas was supplied until the pressure inside became 1atm. Then, DC was applied and the sample was exposed to discharge with currents of $2-4 \mathrm{~mA}$ and voltage of $4000-6000 \mathrm{~V}$. The duration time of the discharge was $\sim 60 \mathrm{~min}$.

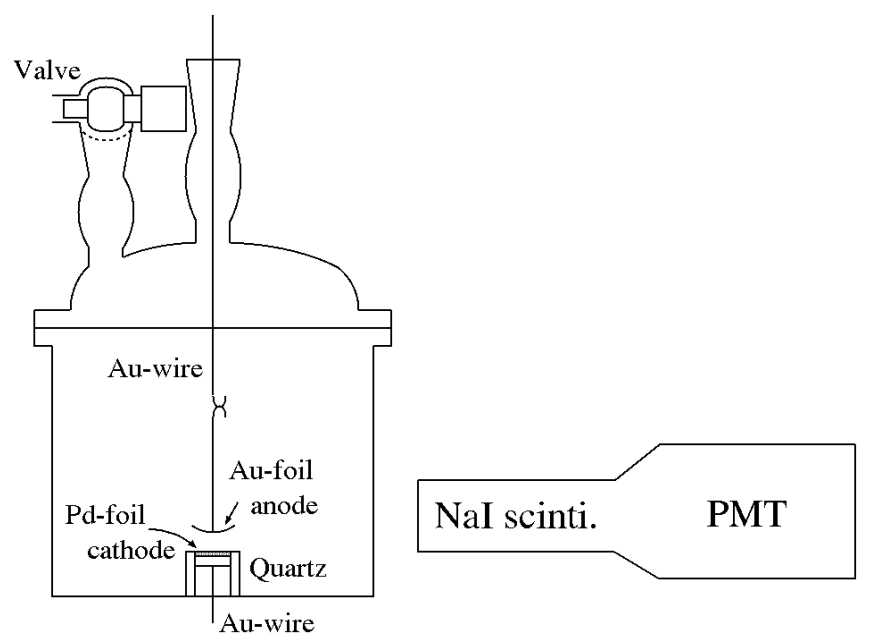

Figure 1. Experimental apparatus.

A NaI scintillation counter (THORN EMI Electron Tubes Ltd.) was used to detect gamma rays emitted from the sample during the discharge. It was placed perpendicular $\sim 10 \mathrm{~mm}$ away from the side wall of the cell. The photons from the NaI counter were detected by a photomultiplier tube (PMT). The bias voltage applied to the PMT was set to $600 \mathrm{~V}$ in this experiment, and the gain was $\sim 10^{4}$ at that biased voltage. The signal from the PMT was amplified and the charge distribution was obtained using a multi-channel analyzer (EG\&G ORTEC), then it was converted to an energy measurement of the gamma rays. The energy calibration for the counter was executed using ${ }^{57} \mathrm{Co}$ and ${ }^{137} \mathrm{Cs}$, which emit gamma rays with the energies of $122.1 \mathrm{keV}$ and $661.7 \mathrm{keV}$ respectively. The energy resolutions were estimated to be $\sim 25 \mathrm{keV}$ and $\sim 80 \mathrm{keV}$ (FWHM) for both energies.

For some samples, the surface was analyzed by time-of-flight secondary ion mass spectroscopy (TOF-SIMS) (ULVAC-PHI:TFS-2100) after the discharge. TOF-SIMS has good sensitivity for detecting a small amount of elements on the surface, and high mass resolution, which allows us to distinguish most atomic ions from the fragmented ones with the same nominal masses. In addition, TOF-SIMS was capable of analyzing all the elements with their isotopes. However, it is difficult to show the results of quantitative analysis from TOF-SIMS results alone. Therefore, in this study, the possibility of new element production and anomaly in the isotopic abundance for the elements detected was only investigated, not confirmed. 


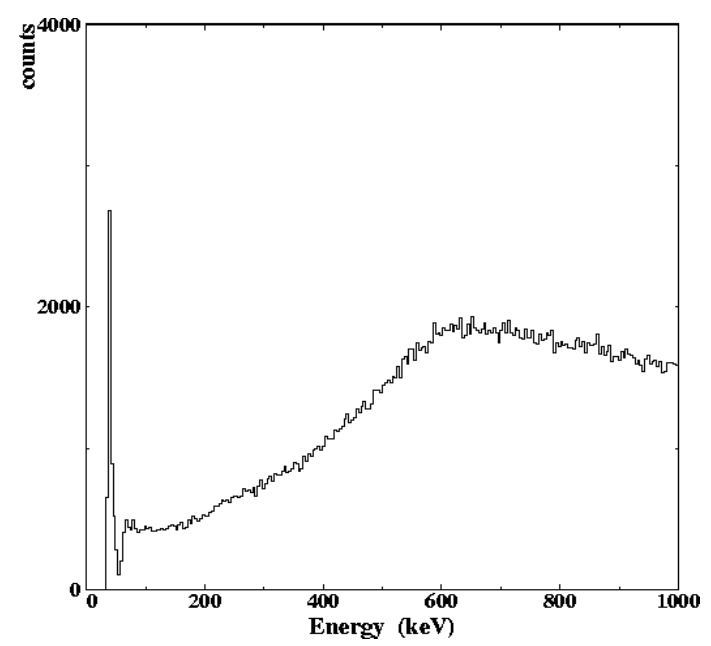

Figure 2. Gamma ray spectrum for the background run.

\section{RESULTS AND DISCUSSION}

\subsection{GAMMA RAY MEASUREMENT}

Figure 2 shows the energy spectrum for a background run measured without the usual glow discharge procedure. For most runs in which the deuterated Pd sample was exposed to discharge in deuterium atmosphere the energy spectra were quite similar to that for the background run. However, the anomalous signals in the gamma ray spectra with energies of 100-200 keV sometimes appeared. Such signals were observed in 10 out of 50 runs. The spectra for these 10 runs were shown in Figure 3. Note that the height for the dark current signal (the peak for the lowest energy for each distribution) was significantly lower from sample- 6 to sample- 10 because the recalibration for the detector system was done between the runs for sample-5 and sample-6. By observing the spectrum on an on-line monitor, the anomalous signals were found just after applying the DC power and were continuously observed during the discharge. If these signals were caused by the electric noise introduced by the discharge, they should have appeared more frequently. Thus, it is assumed that some nuclear reactions that produce the radioactive source took place during the experiment and it emitted the gamma rays. In a previous glow discharge experiment with an inside cell pressure of $\sim 3$ Torr, we observed similar gamma emissions in 4 out of 105 runs [8]. Comparing the results obtained under glow and glow-like discharge conditions, the reaction is more likely to take place under latter case. Therefore, the discharge under a significantly higher density deuterium environment is expected to induce the reaction more effectively, and it would be quite interesting to see what happens with much higher deuterium pressure. Since our current cell is not designed for a discharge under a pressure of greater than 1atm, new type cell will be prepared for the future study. 


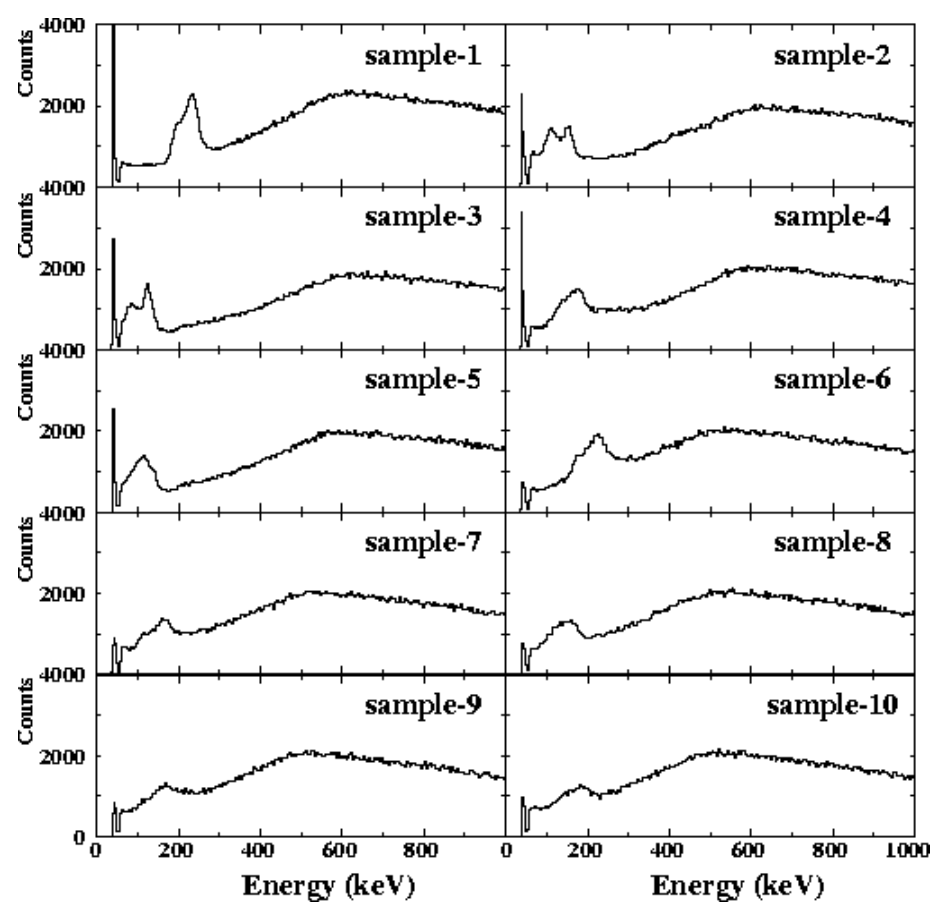

Figure 3. Anomalous gamma ray spectra.

The energy of emitted gamma rays was determined precisely by fitting an appropriate function to the spectrum. In this analysis, a combination of two Gaussian functions and a linear function was chosen to be fitted, considering the energy resolution of the detector. Table 1 shows the mean value and the standard deviation obtained by fitting the two Gaussians to the signal. The emitted gamma ray energy was found to distribute in $80-230 \mathrm{keV}$ regions. Considering the energy resolution estimated from the calibration data, the standard deviations of fitted Gaussians, $\sim 20 \mathrm{keV}$, are supposed to be reasonable in the energy region. 
Table 1. Gamma ray energy obtained from Gaussian fitted, and the standard deviation in parenthesis.

\begin{tabular}{r|r|r|r|r|r} 
& \multicolumn{1}{|c|}{ Sample 1 } & \multicolumn{1}{c|}{ Sample 2 } & Sample 3 & Sample 4 & Sample 5 \\
\hline $\mathrm{E}_{1}(\mathrm{keV})$ & 193.8 & 113.3 & 83.6 & 134.0 & 91.0 \\
& $(10.2)$ & $(15.6)$ & $(15.2)$ & $(14.6)$ & $(25.3)$ \\
$\mathrm{E}_{2}(\mathrm{keV})$ & 230.2 & 153.3 & 124.2 & 172.7 & 124.3 \\
& $(16.6)$ & $(10.6)$ & $(12.5)$ & $(17.7)$ & $(18.5)$ \\
& & & & & \\
& Sample 6 & Sample 7 & Sample 8 & Sample 9 & Sample 10 \\
\hline $\mathrm{E}_{1}(\mathrm{keV})$ & 170.1 & 117.5 & 132.1 & 165.1 & 137.9 \\
& $(17.0)$ & $(13.0)$ & $(23.6)$ & $(21.5)$ & $(20.4)$ \\
$\mathrm{E}_{2}(\mathrm{keV})$ & 219.1 & 162.6 & 164.8 & 215.6 & 184.5 \\
& $(24.5)$ & $(19.0)$ & $(13.0)$ & $(12.9)$ & $(20.2)$
\end{tabular}

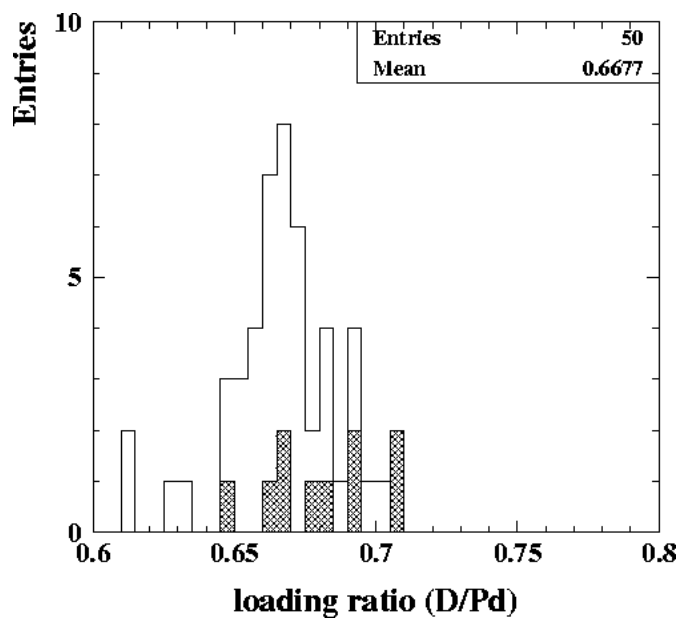

Figure 4. Loading ratio $\mathrm{D} / \mathrm{Pd}$. The hatched histogram is for the sample in which anomalous gamma rays were observed.

Any anomalous signal was not seen for the Pd sample that was not loaded in advance (the no-loading sample). We also carried out a discharge experiment with a hydrated Pd cathode in hydrogen atmosphere in 50 runs. We did not observe any anomalous peak in the gamma ray spectrum, such as the ones we observed in the deuterium experiment. This is additional evidence that the anomalous signals in the deuterium experiment were not due to noise.

Figure 4 shows the distribution of the $\mathrm{D} / \mathrm{Pd}$ ratio for all 50 samples. The hatched histogram shows one for the samples in which anomalous gamma ray signals were observed. Although it has been claimed that there is a strong correlation between the reaction rate of the nuclear reaction in solids and the deuterium loading ratio, no clear evidence for this was found. It is supposed that a threshold for the loading ratio for effectively inducing the reaction is much higher.

Let us consider a model that has been proposed to explain the production of radioactive sources that emitted gamma rays during the discharge. One of the possible explanations for 
the formation of radioisotopes in our experimental condition is the low-energy photofission (LEPF) of Pd proposed by Takahashi et al. [10]. They claim that a high peak flux of low-energy photons in a dynamic lattice of Pd deuteride (hydride) can lead to the fission of $\mathrm{Pd}$ via a selective channel. Their model is in good agreement with some experimental results such as Z-distribution, mass distribution and isotopic ratios. Although the origin of such a photon source is still to be studied, a dynamic condition in our experiment, that is, exposing Pd deuteride to glow-like discharge, might generate high-flux photons with $<50 \mathrm{keV}$ and satisfy the critical condition required to induce LEPF. In the model, major fission products of the Pd are stable isotopes, however there exist some channels that produce short-lived radioisotopes. ${ }^{51} \mathrm{Ti}(5.8 \mathrm{~m}),{ }^{52} \mathrm{Ti}(1.7 \mathrm{~m}),{ }^{52} \mathrm{~V}(3.8 \mathrm{~m}),{ }^{55} \mathrm{Cr}(3.5 \mathrm{~m}),{ }^{56} \mathrm{Cr}(3.6 \mathrm{~m})$ and ${ }^{56} \mathrm{Mn}(2.6 \mathrm{~h})$ are predicted to be produced in significant amounts. Among them, ${ }^{52} \mathrm{Ti}$ and ${ }^{56} \mathrm{Cr}$ can emit $124 \mathrm{keV}$ and $83 \mathrm{keV}$ gamma rays, respectively, in their beta decay process. Thus, the gamma rays observed around $80 \mathrm{keV}$ and $120 \mathrm{keV}$ might correspond to them. However, the gamma rays in the $150-230 \mathrm{keV}$ regions are not emitted from the radioisotopes mentioned above. We may consider the following two crude assumptions: 1) there is another fission channel of $\mathrm{Pd}$ to produce the radioisotopes which can emit $160-230 \mathrm{keV}$ gamma rays. In this case, introducing a new process or modifying the LEPF model is required; 2) $\mathrm{Pd}$ is transmuted (in whichever reactions, fusion or fission) to other stable isotopes, then it fissions to radioisotopes by the LEPF process.

Another consideration is necessary for the results in $\mathrm{Pd}-\mathrm{H}$ experiment. As described above, no anomalous gamma emission was observed for Pd hydride sample. If high-flux low-energy photons are generated around the cathode, in principle, the LEPF can be induced even in hydrogen system. Actually the fission-like transmutation has been reported in some experiments in the hydrogen environment, for example, plasma electrolysis in light water [11]. However, as long as our experimental method, deuterium is assumed to play an important role in inducing a low-energy nuclear reaction.

\subsection{SURFACE ANALYSIS}

For some Pd samples including both types that emitted gamma rays during the discharge ("gamma sample") and did not ("no-gamma sample"), the sample surface was analyzed by the TOF-SIMS. The element composition of the two types was compared. Also, the composition for the Pd sample exposed to the discharge was compared with that of fresh Pd. In this analysis, $\mathrm{Ga}^{+}$was used as primary induced ion. The TOF-SIMS is equipped with a sputtering feature, with a continuous Ga ion beam to clean the sample surface. The feature also gives depth profile of elements distribution on each layer, by changing duration time of sputtering. We analyzed some randomly selected areas (40 microns square) of each sample with sputtering for $0 \mathrm{sec}$ (no sputtering) and $10 \mathrm{sec}$. After the mass spectrum was obtained, candidates of an element in the mass spectrum were selected by considering the mass value of fragmented molecular compounds. Since it is difficult to estimate quantitatively how much a specified element increase or decrease in an area by the TOF-SIMS alone, our analysis was concentrated on checking the existence of some particular elements and their isotopic abundance. We investigated 8 samples (4 out of 8 are "gamma-samples") and 5 fresh samples and compared them. As a result, we did not find clear evidence that any new elements were produced during the experiment. In our glow discharge experiment (i.e. discharge under $\sim 3$ Torr deuterium pressure), Be had been found to be a candidate of a nuclear product [9], but it was not detected in this analysis. According to the TOF-SIMS results, the major impurities of the Pd sample were $\mathrm{K}, \mathrm{Ca}, \mathrm{Ni}, \mathrm{Cr}, \mathrm{Fe}$ and $\mathrm{Cu}$. The isotopic abundances for these elements were checked, and no anomaly was found; the isotopic abundance for these elements was in good agreement with natural one. If a nuclear reaction such as the LEPF really happened during the experiment, changes in isotopic abundance from natural ones should be found in the surface analysis. In fact, in the prediction by the LEPF model, some isotopes of $\mathrm{Ca}, \mathrm{Cr}, \mathrm{Fe}$, $\mathrm{Cu}$ and so on can be produced selectively by the fission of $\mathrm{Pd}$ isotopes. However, we did not find any such anomaly. It is possible that a very small amount of the elements were produced in this experiment and the yield was below sensitivity of the TOF-SIMS. So some interesting facts might be still hidden underground. We should keep investigating the nuclear products carefully with various methods. 


\section{CONCLUSION}

We observed gamma rays in $80-230 \mathrm{keV}$ region during DC glow-like discharge with a deuterated Pd electrode in a deuterium atmosphere. Some short-lived radioisotopes were probably produced in a low-energy nuclear reaction. The efficiency for observing such gamma emissions was enhanced by increasing the pressure of the atmospheric gas in the discharge, and this indicates that the efficiency can be more improved under much higher pressures. This is an interesting task for the future study. The LEPF can take place in our experimental condition and it explains the formation of short-lived radioisotopes, which can be source of gamma emission. Some of our results in gamma ray observation agree with the prediction obtained using the LEPF process. On the surface analysis, we did not obtain clear evidence of nuclear reaction. We will continue the elemental analysis with various analysis methods. These studies can help understand a mechanism of a low-energy nuclear reaction in condensed matter.

\section{Acknowledgements}

We would like to extend our appreciation to Professor A. Takahashi (Osaka University) for helpful comments on this study.

\section{References}

1. I.B.Savatimova et al., Fusion Technol, 26 (1994) 389.

2. X.Z.Li et al., Fusion Technol, 26 (1994) 384.

3. J.Dufour et al., Fusion Technol, 26 (1994) 375

4. A.B.Karabut, Proceedings of 9th International Conference on Cold Fusion, (2002) 155.

5. I.B.Savatimova et al., Proceedings of 9th International Conference on Cold Fusion, (2002) 312.

6. J.Dash et al., Proceedings of 9th International Conference on Cold Fusion, (2002) 77.

7. A.G.Lipson et al., Proceedings of 9th International Conference on Cold Fusion, (2002) 208.

8. H.Yamada et al., Fusion Technol, 39 (2001) 254.

9. A.Arapi et al., Jpn. J. Appl. Phys., 41 (2002) L1181.

10. A.Takahashi et al., Jpn. J. Appl. Phys., 40 (2000) 7031.

11. T.Ohmori, Topics in Electrochem., 7 (2000) 101. 